\title{
Capsule Commentary on Weeks et al., Measuring Primary Care Organizational Capacity for Diabetes Care Coordination: The Diabetes Care Coordination Readiness Assessment
}

\author{
Lizheng Shi, PhD, MSPharm ${ }^{7}$ and Anjum Khurshid, PhD, MBBS, MPAff ${ }^{2}$ \\ 'Department of Global Health Systems and Development, School of Public Health and Tropical Medicine, Tulane University, New Orleans, LA, USA; \\ ${ }^{2}$ Louisiana Public Health Institute (LPHI), New Orleans, LA, USA.
}

J Gen Intern Med 29(1):178

DOI: $10.1007 / \mathrm{s} 11606-013-2614-y$

(c) Society of General Internal Medicine 2013

$C$ are coordination for chronic disease is an important $\mathcal{C}$ strategy for managing costs while improving quality. Weeks et al. ${ }^{1}$ describe a tool to help primary care practices measure their readiness to provide effective care coordination for adult type 2 diabetes. The Diabetes Care Coordination Readiness Assessment (DCCRA) is a self-assessment guide to identify barriers and actionable areas to improve diabetes care coordination services, and measures five domains: organizational capacity, diabetes care coordination best practices, clinical management capability, quality improvement capability, and technical infrastructure. The DCCRA had excellent internal consistency (Cronbach's alpha 0.96), was validated in 39 practices in the Beacon Community of Inland Northwest (BCIN), and reliably discriminated among practices on the five domains of diabetes care coordination. The DCCRA can be improved by item reduction in the future. There are a couple of challenges to the implementation of the DCCRA. First, self-reported data collection without any in-depth assessment of the capabilities identified in the DCCRA may not give an accurate picture of a practice's actual capacity. Often, practices equate having the capability of disease management or care coordination in their electronic medical record systems with their ability to perform those tasks routinely. Effective care coordination, just like any other clinical improvement effort, requires an in-depth analysis of other aspects, such as culture, workflow, leadership readiness, and team motivation, in order to come up with meaningful ways of achieving it. ${ }^{2}$ Second, the DCCRA as a diabetes- specific care coordination assessment system should be further validated by the nationally recognized patient-centered medical home (PCMH) approach, ${ }^{3}$ which includes care coordination as an important part of establishing a patient-centric, accountable, and efficient system of care. ${ }^{4}$ The DCCRA is an important step in helping practices demonstrate capacity for care coordination in diabetes, but additional research is needed to demonstrate that this capacity translates into effective care coordination, a process measure, and more importantly, into better patient outcomes.

Conflict of Interest: The authors declare that they do not have a conflict of interest.

Corresponding Author: Lizheng Shi, PhD, MSPharm; Department of Global Health Systems and Development, School of Public Health and Tropical Medicine Tulane University, 1440 Canal Street Suite 1900, New Orleans, LA 70112, USA (e-mail: lshi1@tulane.edu).

\section{REFERENCES}

1. Weeks DL, Polello JM, Hansen DT, DC, Keeney BJ, Conrad DA. Measuring primary care organizational capacity for diabetes care coordination: the diabetes care coordination readiness assessment. J Gen Intern Med. doi:10.1007/s11606-013-2566.2.

2. Wise CG, Alexander JA, Green LA, Cohen GR, Koster CR. Journey toward a patient-centered medical home: readiness for change in primary care practices. Milbank Q. 2011;89(3):399-424. doi:10.1111/j.14680009.2011.00634.x.

3. Arend $\mathbf{J}$, Tsang-Quinn $\mathbf{J}$, Levine $\mathbf{C}$, Thomas $\mathbf{D}$. The patient-centered medical home: history, components, and review of the evidence. Mt Sinai J Med. 2012;79(4):433-450. doi:10.1002/msj.21326. Review.

4. Peikes D, Zutshi A, Genevro JL, Parchman ML, Meyers DS. Early evaluations of the medical home: building on a promising start. Am J Manage Care. 2012;18(2):105-116. Review. 